Research Note

\title{
The Idea of Progress: A Theoretical and Concise Goal- Structure Model
}

\author{
${ }^{1}$ Rodolfo Henrique Cerbaro and ${ }^{2}$ Jamie Lyn Whisler \\ ${ }^{I}$ School of Economic, Administrative and Accounting Sciences, University of Passo Fundo, Passo Fundo, Brazil \\ ${ }^{2}$ Business Division, Bay de Noc Community College, Escanaba, Michigan, USA
}

\author{
Article history \\ Received: 04-06-2016 \\ Revised: 02-08-2016 \\ Accepted: 04-08-2016 \\ Corresponding Author: \\ Rodolfo Henrique Cerbaro \\ School of Economic, \\ Administrative and Accounting \\ Sciences, University of Passo \\ Fundo, Passo Fundo, Brazil \\ Email: sengicerbaro@gmail.com
}

\begin{abstract}
The idea of progress as it currently stands is not well understood. This is because it is taken to be both context-dependent and stands independently, without a formal structure. It is an idea which is applied in cases that can be as diverse as simply finishing an essay to the complex progress of humanity. If the idea appears in such diverse scenarios, is there an underlying mechanism through which we can easily understand it? Here we structure the idea and propose a simple postulate which allows us to have a more adequate knowledge of it. The expected result is the capability of realizing it's intellectual appearance and understanding it in any given practical scenario; even if it is not totally clear based on what we define as progress. This short and simple model implies that the action of a goal definition should be handled with care, as the concept of progress is engulfed by the goal system.
\end{abstract}

Keywords: Idea of Progress, Goals, Intellectual Structure of Progress

\section{Introduction}

What is progress? An unresolved issue to Malthus (Levin, 1966), progress is defined as "a forward or onward movement (as to an object or a goal)" or a "gradual betterment" (Merriam-Webster.com, 2015). This is somewhat vague and furthermore the literature about progress (Bury, 1987; Nisbet, 1980; Lange, 2011) has dealt with the idea independently of a structure. If understood as such, we then face difficulty in making sense of it, because scenarios as diverse as a loading screen advancing to driving to a given destination or to gaining a promotion all have the idea of progress within them. This diversity causes difficulty in making sense of what truly can be considered as progress and is completely context-dependent. Not only it is dealt in free-form in the literature, but it is also only applied to matters such as the "progress of humanity" (Gordon, 2011; Marx and Mazlish, 1996) or the "progress of science" (Bird, 2007), that is, broad goals, therefore it appears unrelated with daily life affairs, which is an incomplete view, considering it is one of the most important ideas permeating everyday life. Progress provides incentives for actions. Without the reward of progress, what would be the point in achieving any set goal? As diverse as progress may be, there needs to be a common thread that ties the entire idea together.
We propose here that, as diverse as the idea may appear, it is in fact quite a simple structure we need in order to understand it. First we propose a formal structure of progress and two setbacks it has, then we will apply it in practical scenarios in order to realize it's functionality. The postulate which follows also allows falsifiability, because within each practical scenario the subject on which the idea of progress is understood changes, according with the goal and therefore one can consider it as correct or incorrect. Also, in some cases we cannot even properly specify what it is in practice, but knowing the structure allows us to understand why we are applying the idea of progress to the specific case.

It is hoped that we achieve a broad understanding of the idea and how it permeates many scenarios. Although it is possible to have an intuitive understanding without a structure, it is knowing the structure that allows us a functional knowledge regarding the idea and to be more careful as to how and towards what we are applying it to. With such knowledge, we can adapt to an ever-changing landscape of goals being defined, creating the idea of progress. This idea of progress provides incentives for actions and permeates everyday life, from our main life goals to even the most mundane ones, therefore having a structure to deal with it in mind is a useful and significant model to routinely apply, as we realize just 
how important it is to adequately define our goals and the goals of our institutions.

Considering the idea of progress as a psychological structure is a different approach, as it has not been treated as being so in the literature; what we have are attempts to theorize the idea which do not consider psychology and only envision the idea as applied to a select few, broad goals. Our structure, therefore, comes as a generalization of the idea, providing us a tool to understand the idea as applied to many scenarios and, as such, being of use to a wide range of situations and professionals.

\section{A Structure Concerning the Understanding of the Idea of Progress}

To understand the idea of progress we must first define the concept of a goal, that is, consider a goal to be the purpose toward which an endeavor is directed. Then we have that: progress is what counts as a means of advancing towards the end result of a given defined goal. Although incredibly simple, as we will see in applying this postulate, it seems to be a functional way of understanding the idea of progress. This also gives us the ability to measure progress or conversely, regress.

There are two setbacks which appear with such a structure. First is that one must be able to define a goal, otherwise defining what counts as a means of advancing towards the end result of a given defined goal is not possible and progress not achievable. Take for instance the idea of the progress of humanity-there are several competing subjects on which the postulate is based, ranging from the advancement of science, to improved quality of life or to environmental improvements, among others. The structure for the comprehension remains the same, but the solution for the scenario depends on categorization and, hence, controversy can arise. This model does not solve the problem however, as defining the goal is a necessary condition for us to properly define progress in the scenario. If there is controversy regarding what goal should be used, there will be controversy as to how the idea of progress is comprehended.

The second setback is that there are goals where defining what counts as a means of advancing towards the end result of a given defined goal is not crystal clear, usually (but not solely) because the goal is not specified well enough. For example, consider the goal of passing a test: progress can be taken one of a variety of ideas such as the necessary time spent studying to pass, the time lapse between the test and the results being released or the physical action of writing the answers. Sometimes all that is required is writing down or envisioning the goal in a more specific way, but in other cases it may be the case that the goal is complex enough to accept several means of advancing towards its end, which does not allow us to pinpoint what the idea of progress is based upon.
These two setbacks, however, do not make the understanding of the structure less important, because in many scenarios the model functions well and it allows us to understand how we are operating regarding the idea of progress. Paying attention to the frequency that notions of progress appear is a useful task, as one concludes daily life is permeated by these notions, which help us understand how we are operating, but the literature has thus far not presented any model regarding the psychological structure of the idea.

\section{Linearly Applying the Model}

Consider a person concerned with the goal of attaining more wealth, then in applying the postulate we find that attaining wealth is "what counts as a means of advancing towards the end result of the given defined goal", hence attaining more wealth equals progress and, losing wealth, regress. Now let us briefly change the goal from attaining more wealth to competing with an individual for wealth. This automatically changes the idea of progress, from attaining more wealth to attaining more relative wealth compared to a given individual. In this scenario, even though the person can have more wealth in relation to the past, which was the first goal mentioned above, they may not have achieved their goal. It can be that the difference in wealth of the other person has increased in relation to the subject creating regress. The second goal set the structure where the subject had to create more relative wealth to be able to progressmeaning that even with the same result in achieving more wealth, may not always progress towards the goal, depending on the definition.

So progress morphed from being attaining more wealth to attaining more wealth compared to a given individual. The postulate allows the idea of progress to morph without us losing the understanding of it. It should be noted, the goal is simple enough for us to use the model, as long as we understand the defined goal. Such happens in many scenarios, making it imperative one is clear in their understanding towards which subject the idea of progress is concerned.

\section{Plurality of Means of Advancing Towards the End Result of a Goal}

As we mentioned above, there can be a variety of means of "progress" that act as components in achieving a goal. Take a novice baker with the goal of baking a cake. This goal accepts various means of advancing towards its end-obtaining the ingredients, preparing the recipe, the process of baking-therefore the model can be applied and allows us to have an intellectual understanding of what is being done. However specifying what we use as a basis of establishing the idea of progress is difficult, as there are a plurality of means 
in attaining the result. Even a goal which appears simple, such as drinking water, has a plurality of means of advancing towards its end: progress can be understood as moving the necessary distance to attain the water, filling a cup or even opening the mouth in order to drink.

However, a complex goal does not diminish the achievement of progress. As one moves through life, they encounter mostly complex goals. A person with no knowledge of baking a cake would not in most cases give themselves the goal of creating the most elaborate wedding cake straight away. Instead they would create interim goals that would be seen as marks of progression. Even if one does not move directly to their main goal, they are not spinning their wheels, they are just checking off sub-goals while slowing moving towards a bigger one, which is still defined as progress. The same could be said for more nonlinear progress. A college student may change paths, or be forced to study subjects not needed for their goal or course of study in order to graduate. They may be pushed off track of their main goal while still making progress in smaller goals necessary to achieve the main goal.

What we see is that although the postulate stands, in some scenarios it remains complicated, however an understanding of the structure allows us to understand why we are applying the idea of progress to the matter at hand. This knowledge is important, because it is not a common human characteristic to think about which goal, exactly, is defined and how one is handling the idea of progress.

\section{Using the Model}

The present model can be used by a vast array of professionals, with the purpose of comprehending the way they operate toward their goals and, consequently, how the idea of progress is handled. Considering an area such as business, it is a fact that organizations have goals which are sought and this model allow us to comprehend what can be considered as progress to the enterprise and how it can change with the way the goal is defined. Scientists usually believe in the idea of progress and there is reason to do so, considering the goals usually defined by them. Even the most skeptic person toward the idea will find that within a given intellectual structure, it seems to be an existing phenomenon. The psychological manifestation of the idea of progress has hitherto not been explored and we intend to show that it is an idea which affects us all, because we are constantly defining goals and progress seems to be best understood within a goal structure scenario. From a person with the goal of simply learning a given discipline to the intellectual worried about whether humanity is moving in a desirable direction, progress is a conceptual thought affecting us constantly and knowledge of the structure comes as a means of aiding us comprehend how our psyches seem to be operating.

Although questions of whether progress is solely an intellectual idea or if it exists outside of intellects remain unanswered, using of this model comes as a new thought regarding how the idea of progress is interpreted, from not being linked to psychology to finding its basis through a psychological structure. Linking this idea with psychology provides us a novel way of framing progress, one we can carry with us in our daily lives and comprehend how we apply it in our personal and professional affairs.

\section{Conclusion}

A goal-structure model was built in order for us to understand the idea of progress. The structure and two important setbacks were discussed. This appears to be the simplest possible way of understanding the idea of progress, because it simply relates it with the definition of a goal and allows for an all-encompassing understanding of all possible practical scenarios where the idea of progress is present. Considering that whenever we have a goal, there are one or more ways of advancing towards the end result, we realize that the idea of progress is ever present in our intellects, acting as a tool which aids us to understand why we do what we do.

It has been argued that no other idea has been more influential than the idea of progress (Nisbet, 1980) and with reason, because we hope our efforts lead us somewhere rather than being in vain. The literature has also dealt with the notion of progress as applied to broad goals, such as the progress of the human race (Lange, 2011). What this model does differently is show that progress is an intellectual structure, also appearing when goals which are not directed to the common good are followed. This implies that knowing such a structure and being careful is necessary, as observers using certain structures can have in their minds that they are progressing, but these ideas might collide with more ethical, noble goals. Take for example a logging company-if their goal is to profit from deforestation, this collides with a global goal of environmental protection. However, progress can be self-defined and self-observed or it can be viewed by an outside observer-what is considered as progress for one person may not be for another and yet it is still progress considering the intellectual structure.

It is also important for individuals to understand such a structure in order to not stay trapped within goals that are unlikely to accomplish anything: for example, a person who has long had the goal of advancing in a game may keep applying the idea of progress to the game, instead of applying it to matters which can be more useful for society. The idea of progress is a dangerous one, as it is the main driver of individuals once they have defined which goals they are seeking, therefore much effort may be undertaken in order to progress. Understanding the structure allows us to intellectually change goals and, consequently, comprehend progress differently. It also makes us conscious that it is an intellectual structure given that a goal, or set of goals, is defined. 


\section{Acknowledgement}

Rodolfo Henrique Cerbaro would like to dedicate the work to his mother, Ignes Cerbaro.

\section{Funding Information}

The authors have no support or funding to report.

\section{Author's Contributions}

Rodolfo Henrique Cerbaro thought of the model and wrote parts of it; Jamie Lyn Whisler helped with the writing and the way it is presented.

\section{Ethics}

This article is original and contains unpublished material. The corresponding author confirms that all of the other authors have read and approved the manuscript and no ethical issues involved.

\section{References}

Bird, A., 2007. What is scientific progress? Noûs, 41.1: 64-89. DOI: 10.1111/j.1468-0068.2007.00638.x

Bury, J.B., 1987. The idea of Progress: An Inquiry into its Origin and Growth. 1st Edn., Courier Corporation, ISBN-10: 0486254216, pp: 357.
Gordon, D., 2011. The Turgot Collection: Writings, Speeches and Letters. 1st Edn., Ludwig von Mises Institute. ISBN-10: 1479361038, pp: 560.

Lange, M.M., 2011. "Progress", The Stanford Encyclopedia of Philosophy (Spring 2011 Edition). E.N. Zalta (Ed.).

Levin, S.M., 1966. Malthus and the idea of progress. J. History Ideas, 27: 92-108. DOI: $10.2307 / 2708310$

Marx, L. and B. Mazlish, 1996. Progress: Fact or Illusion? 1st Edn., University of Michigan Press, Ann Arbor, ISBN-10: 0472106767, pp: 232.

Nisbet, R.A., 1980. History of the Idea of Progress. 1st Edn., Transaction Publishers, ISBN-10: 1412825482, pp: 370 .

Merriam-Webster.com, 2015. "Progress". MerriamWebster.com. 2015. http://www.merriamwebster.com 